\title{
1 Marktplätze in der Pflege - Konsolidierung und Effizienzsteigerung durch Digitalisierung
}

\author{
Maximilian Greschke
}

Erfolgreiche Unternehmen wie Uber oder Airbnb aus den USA haben mit digitalen Marktplätzen einst stark fragmentierte Märkte wie Taxi- oder Unterkunftsvermittlung binnen weniger Jahre erfolgreich konsolidiert. Und spätestens seitdem aus diesen Vorhaben in kürzester Zeit milliardenschwere Unternehmen entstanden sind, entstehen überall auf der Welt ähnliche Plattformen zur Lösung vergleichbarer Probleme.

Nun scheint es so, als wäre diese Zeit auch für die Pflege gekommen. Von 2014 bis 2015 haben sich Venture Capital Investments im Care Coordination Bereich mehr als verdoppelt und bilden mit fast 230 Millionen Euro die mittlerweile zweitgrößte Investmentsparte im Digital Health Bereich. Im Vorjahr 2014 lag Care Coordination mit einem Volumen von rund go Millionen noch auf Platz 3 hinter Life Science Investments (Rock Health 2015). Drei besonders prominente Beispiele dieser Entwicklung im Bereich der Marktplätze für Pflege sind die drei amerikanischen Unternehmen Honor, Home Hero und Hometeam, welche gemeinsam fast 130 Millionen USD an Investment einsammelten.

Da ist es wenig verwunderlich, dass sich diese Entwicklung nun auch in Europa abzeichnet. Allein in Deutschland wurden seit 2015 mittlerweile sieben Plattformen mit dem Ziel gestartet, einen Marktplatz für ambulante Pflegeleistungen zu etablieren. 


\section{Warum ist das Marktplatzmodell für die Pflege in Deutschland attraktiv?}

Laut der Pflegestatistik 2015 des statistischen Bundesamtes betreut der durchschnittliche ambulante Pflegedienst in Deutschland derzeit nur 52 Patienten. Insgesamt haben nur 2,8\% aller ambulanten Dienste Zugriff auf mehr als 150 Patienten. Insgesamt haben die zehn größten ambulanten Pflegedienste in Deutschland weniger als $2 \%$ Marktanteil. Das Potenzial für Konsolidierung ist also enorm. Der stationäre Bereich ist leicht schwächer fragmentiert. Marktplätze können im ambulanten Bereich jedoch mehr Probleme lösen.

Eine kleine Verzerrung der Statistik findet wohlgemerkt dadurch statt, dass freigemeinnützige Träger, wie zum Beispiel kirchliche Werke, durch Landesund Ortsverbände organisiert sind. Diese profitieren von einer gemeinsamen Marke, aber haben darüber hinaus keine gemeinsamen operativen Skaleneffekte vorzuweisen.

In Märkten mit einer starken, lokalen Fragmentierung entstehen klassische Problemfaktoren für Endkunden, welche ein Marktplatz gut lösen kann: Woher weiß ich, welcher Pflegedienstleister wirklich gut ist? Welcher ist der richtige für mich? Bekomme ich wirklich alles, was mir an Leistungen zusteht (wird fair abgerechnet?). Somit ist die Grundvoraussetzung geschaffen.

Marktplatzmodelle sind häufig durch Risikokapital finanziert und somit darauf ausgelegt, schnell signifikante Marktanteile zu gewinnen. Die technologischen Herausforderungen eines Marktplatzes sind gleichzeitig (zumindest am Anfang) eher trivial und entsprechend leicht ist eine junge Plattform nachzuahmen.

Essenziell für die Umsetzung und einen attraktiven Return on Investment sind deshalb Skaleneffekte. Bei einem zweiseitigen Marktplatz bezeichnet man diese auch als Netzwerkeffekte. Ein solcher Netzwerkeffekt tritt dann auf, wenn ein zusätzlicher Anbieter oder Nachfrager auf dem Marktplatz den Wert des Marktplatzes für die jeweils andere Seite steigert (s. Abb. 1).

Wenn wir darüber nachdenken, ob solche Netzwerkeffekte auch im Pflegemarkt auftreten, müssen wir zuerst die Seite der Pflegekräfte betrachten.

In Deutschland werden ungefähr ein Drittel aller zuhause gepflegten Pflegebedürftigen durch einen professionellen Pflegedienst versorgt, zwei Drittel hingegen von pflegenden Angehörigen und deren privaten Unterstützern (Statistisches Bundesamt 2017).

Für die Erbringung von Behandlungspflege (also medizinischen Leistungen) im Rahmen des Pflegedienstgeschäftes müssen gut ausgebildete Pflegekräfte angestellt werden. Bereits heute herrscht in dieser Berufsgruppe jedoch ein immer stärker werdender Fachkräftemangel. Es ist davon auszugehen, dass alle Bemühungen des Bundes diesen nicht verhindern können, da die demografische Entwicklung den Bedarf stetig nach oben treibt. Entsprechend begrenzt ist das Angebot an Pflegekräften in einem lokalen Markt. 


\section{Nachfrage profitiert von mehr Angebot}

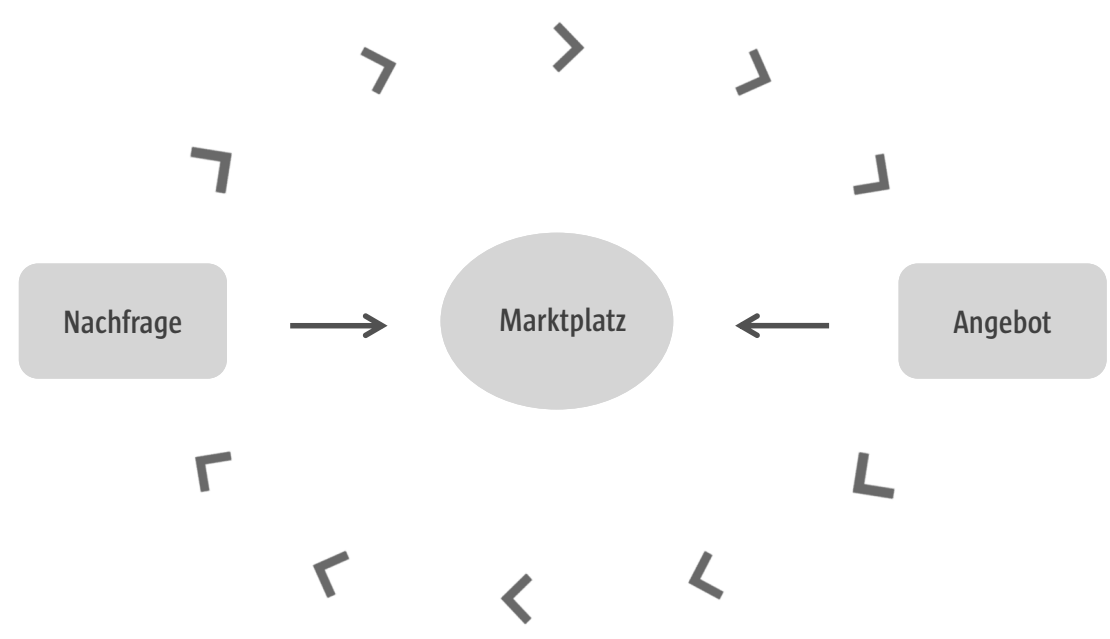

Angebot profitiert von mehr Nachfrage

\section{Abb. 1 Netzwerkeffekt}

Dadurch ergibt sich eine Marktdynamik, für welche die folgenden Punkte immer zutreffen:

- In jedem lokalen Pflegemarkt ist die Anzahl der Pflegebedürftigen größer, als die der Pflegekräfte.

- Eine Pflegekraft hat mehr Kontakt (da mehr Arbeit mit mehreren Patienten) mit einem Pflegeanbieter, als eine pflegebedürftige Person.

- Häusliche Pflege ist ein zweiseitiger Markt - es gibt also genau gesagt zwei Seiten, auf denen Pflegeanbieter miteinander im Wettbewerb stehen - und somit auch zwei Seiten auf denen eine Winner-takes-it-all-Dynamik entstehen kann.

Die Art und Weise, wie die beiden Marktplatzseiten miteinander interagieren, erzeugt eine Winner-takes-it-all-Dynamik. Das ist ein Fall, indem ein besonders starker Netzwerkeffekt vorhanden ist.

Wenn wir uns vorstellen, dass es einem Marktteilnehmer gelingt, eine Mehrzahl an Pflegebedürftigen, also Nachfrage, für sich zu gewinnen, trifft folgende Situation zu:

- Dieser Marktteilnehmer verfügt über die höhere Nachfrage.

- In der Gesamtbetrachtung werden Pflegekräfte also mehr Pflegestunden für diesen Anbieter leisten.

- Mehr Pflegekräfte heißt auch, höhere Liquidität auf dem Marktplatz - die wichtigste Kennzahl, um einen gut funktionierenden Marktplatz zu bieten.

- Hohe Marktplatzliquidität wiederum führt zu besserem Service (z.B. bessere Verfügbarkeit, geringere Distanz) und dadurch im Umkehrschluss zu höherer Attraktivität für neue Kunden. 
Im Umkehrschluss heißt das für den „Verlierer“ des Marktplatzes:

- weniger Pflegenachfrage,

- dadurch führt die geringere Liquidität zu Mehrkosten (z.B. größere durchschnittliche Distanzen zwischen Pflegekräften und Pflegebedürftigen zu höherem Effizienzverlust durch Fahrzeiten),

- dies macht die Arbeit unattraktiver für Pflegekräfte, da Margen dadurch verloren gehen und

n Pflegekräfte wandern zum Mehrheitsanbieter ab und bieten ihre Leistungen dort an.

Netzwerkeffekte in lokalen Marktplätzen reisen nicht in andere Städte mit. Der Effekt ist nur dort persistent, wo Nachfrage und Angebot auch tatsächlich existieren. Eine starke Marke und Reputation auf der Seite der Pflegekräfte erleichtert jedoch den Einstieg in neue geografische Regionen, da die Arbeitnehmer- oder Auftraggebermarke von Unternehmen ab einem gewissen Größengrad auf jeden Fall Wahrnehmung auf nationaler Ebene generiert. Insbesondere in sehr engen Communities, so wie es in der Pflege der Fall ist.

\begin{abstract}
Die „Commodity“-Natur der Transaktion
Im Gegenzug zu anderen Märkten, die über einen solchen Marktplatzansatz konsolidiert wurden - beispielsweise der Taxivermittlungsmarkt durch Anbieter wie Uber - hat die „Transaktion“ im Pflegemarkt keine „Commodity“-Natur. Das heißt, die persönliche Beziehung zwischen den Marktteilnehmern spielt eine große Rolle. In der Taxivermittlung hingegen ist das Marktplatzbedürfnis eine Transportdienstleistung von einem beliebigen, „ausreichend guten“ Fahrer.

Dadurch verstärkt sich ein Netzwerkeffekt weiter, da eine Nutzung von mehreren Anbietern durch denselben Kunden wenig zielführend ist. Gleichzeitig ist dies traditionell eine unerwünschte Eigenschaft, da eine starke Beziehung zwischen Angebot und Nachfrage leichter zu einer Umgehung der Plattform beiträgt (und somit auch der Monetarisierung durch die Plattform).
\end{abstract}

\title{
Wie passt das in die ambulante Pflegelandschaft in Deutschland?
}

Auf den ersten Blick erscheint es einleuchtend, dass aufgrund dieser Charakteristiken früher oder später eine Konsolidierung im Pflegemarkt erfolgen wird. Wie und wo kann sich so ein Marktplatzmodell in der Praxis jedoch wirklich durchsetzen?

Gesetzgebung und Arbeitsweise der Krankenkassen machen das Erbringen von SGB5-Leistungen (Behandlungspflege) im Rahmen selbstständiger Arbeit mit dem Patienten selbst (bzw. dessen Vertretung) als Auftraggeber sehr schwer (und in manchen Fällen unmöglich). Dies ist jedoch eine Voraussetzung für einen Marktplatz ohne Anstellungsverhältnisse. Wahrscheinlicher ist deshalb die Konsolidierung über ein Plattformmodell im Segment für SGB-11-Leistungen (Grundpflege) bzw. privaten Pflegehilfen.

Die Ineffizienzen in diesem Marktteil liegen auf der Hand: Wer eine private Pflegehilfe sucht, muss immer noch über lokale Kleinanzeigen gehen oder sich auf Word- 
of-Mouth verlassen. Mit der heutigen Technologiepenetration in der Gruppe der Entscheidungsträger, den erhöhten Pflegegeldern und dem Zugriff auf Entlastungsleistungen ist der Weg für moderne digitale Konsolidierungsmodelle nach dem klassischen Marktplatzvorbild jedoch geebnet.

Ohne Zweifel wird auch die Pflegedienstlandschaft über längere Zeit zusammenwachsen, aufgrund der zahlreichen regulatorischen Herausforderungen wird dies aber zeitlich verzögert erfolgen.

\section{Ausblick}

Deutschland befindet sich bei der Digitalisierung der Pflegebranche noch ganz am Anfang. Plattformen sind nicht der einzige Weg, wie Digitalisierung eine Konsolidierung oder allgemeine Effizienzsteigerung in der Branche. Seitdem Risikokapitalgeber bereit sind, in neue Technologien der Branche zu investieren, gibt es auch in anderen Bereichen wie Software-as-a-Service eine Vielzahl von jungen Unternehmen, die versuchen Probleme von etablierten Anbietern zu lösen. Ob sich eine Plattform durchsetzen kann, wird sich erst innerhalb der nächsten Jahre abzeichnen. Tatsache ist aber, dass diese frische Welle an Innovation essenziell für die Pflegebranche ist. Sie wird helfen, die großen Herausforderungen der nächsten Jahre zu bewältigen und zu guter Letzt vor allem den Patienten und den ausführenden Pflegekräften selbst helfen.

\section{Literatur}

Deutschlandergebnisse, Wiesbaden. URL: https://www.destatis.de/DE/Publikationen/Thematisch/Gesundheit/Pflege/PflegeDeutschlandergebnisse5224001159004.pdf?_blob=publicationFile (abgerufen am 11. März 2017)

Rock Health (2015) Digital Health Funding: 2015 Year in Review. URL: https://rockhealth.com/reports/digitalhealth-funding-2015-year-in-review/ (abgerufen am 14. März 2017)

Statistisches Bundesamt (2017) Pflegestatistik 2015. Pflege im Rahmen der Pflegeversicherung 